\title{
Development of a test system to analyze different hip fracture osteosyntheses under simulated walking
}

\author{
Andreas Lenich ${ }^{1-4, *}$, Samuel Bachmeier ${ }^{1}$, \\ Sebastian Dendorfer ${ }^{1}$, Edgar Mayr ${ }^{2}$, Michael Nerlich ${ }^{3}$ \\ and Bernd Füchtmeier ${ }^{4}$ \\ ${ }^{1}$ Laboratory for Materials Science, University of Applied \\ Sciences Regensburg, Regensburg, Germany \\ ${ }^{2}$ Department of Trauma and Reconstructive Surgery, \\ Clinical Centre Augsburg, Augsburg, Germany \\ ${ }^{3}$ Department of Trauma Surgery, University of \\ Regensburg, Regensburg, Germany \\ ${ }^{4}$ Department of Traumatology, Klinikum Rechts der Isar, \\ Technical University of Munich, Munich, Germany
}

\begin{abstract}
The mechanical complications of osteosyntheses after hip fractures are previously investigated by mostly static or dynamic uniaxial loading test systems. However, the physiologic loading of the hip joint during a normal gait is a multiplanar, dynamic movement. Therefore, we constructed a system to test osteosyntheses for hip fractures under physiologic multiplanar loading representative of normal gait. To evaluate the testing system, 12 femora pairs were tested under 25,000 cycles with two standard osteosyntheses (Proximal Femoral Nail Antirotation/Gamma3 Nail). For angular movement, the varus collapse to cut out $\left(\propto_{\mathrm{CO}}\right)\left(\propto_{\mathrm{CO}}=4.8^{\circ} \pm 2.1^{\circ}\right.$ for blade and $\propto_{\mathrm{CO}}=7.8^{\circ} \pm 3.8^{\circ}$ for screw) was the dominant failure mode, and only slight rotational angle shifts $\left(\propto_{\text {Rot }}\right)\left(\propto_{\text {Rot }}=1.7^{\circ} \pm 0.4^{\circ}\right.$ for blade and $\propto_{\text {Rot }}=2.4^{\circ} \pm 0.3^{\circ}$ for screw) of the femoral head around the implant axis were observed. Angular displacements in varus direction and rotation were higher in specimens reinforced with screws. Hence, the cut out model and the migration directions showed a distinction between helical blade and hip screw. However, there were no significant differences between the different implants. The new setup is able to create clinical failures and allows to give evidence about the anchorage stability of different implant types under dynamic gait motion pattern.
\end{abstract}

Keywords: biomechanical implant test; dynamic multiplanar loading; hip fracture; implant migration; patient specific loading.

\footnotetext{
* Corresponding author: Dr. Andreas Lenich, Department of Trauma Surgery, Klinikum Rechts der Isar, Technical University of Munich, Ismaningerstr 22, D-81675 Munich, Germany Phone: +49-89-41405026

E-mail: lenich@uchir.me.tum.de
}

\section{Introduction}

In the industrial world, the incidence of proximal femur fractures rises as the population ages to virtually epidemic proportions. In 2005, hip fractures caused up to 700,000 deaths [11] in the United States; $72 \%$ of the total treatment costs of 2 million fractures were caused by only $14 \%$ of fractures in the hip region $[3,8]$. The estimated postoperative mortality rate after 6 months is described between $17 \%$ and $20 \%$ [3].

The correlation of age and osteoporosis is well-known [11]. In particular, reduced bone quality and osteoporosis are relevant in the proximal femur, causing increased numbers of fractures in this region and aggravated conditions in treating fractures in the proximal femur [18]. The posttraumatic time of immobilization has a severe influence on the postoperative outcome [7]. The aim of the acute surgical treatment is a stable osteosyntheses that postoperatively allows a full body weight (BW) on the injured extremity. For the stabilization of hip fractures, a wide number of different implants are on the market. In the recent years, extra- and intramedullary osteosyntheses have been implanted due to equal complication rates [17]. While both implant types have been improved in the past, serious implant-related complication rates of 5-20\% are still present $[17,19]$. The reasons that lead to those complications and the dependence of those complications have not been investigated sufficiently. Most studies, however, concluded that implant failure or inaccurate implant positioning leads to major complications [1, 14, 20, 21].

The biomechanical tests for osteosyntheses systems seem to have no standard procedures. Most of the implant systems have only undergone material stability tests and clinical investigations. Clinical studies, however, show only the complications that appeared, and if serious, failures in the implants cause injuries [6, 14, 15, 19]. Most implants are investigated in simplified uniaxial biomechanical loading tests $[9,10,20]$. Several biomechanical studies show relevant approaches to study the mechanisms that are correlated to the implantrelated complication rate. In particular, laboratory studies have simulated implant migration and cut out in a controlled and reproducible manner, but under simplified loading conditions. While early biomechanical studies used static uniaxial loading [5, 6, 9, 10, 20, 22], more recent studies have started to use dynamic uniaxial loading $[6,13]$. Bergmann et al. [2] described the movement of the acting loads during normal walking in three dimensions for the hip joint. However, the complex loading scenario seen in the hip during walking and other daily activities has not been simulated in biomechanical investigations. In particular, the combined axial and torsional loading may play a crucial role in the occurring complications and implant failures [6]. A more physiological approach 
used - the biaxial rocking motion - as used to test total hip arthroplasties, showed clinically relevant results in the evaluation and served to date as a state-of-the-art testing system [6].

The objective of this investigation was to develop a test system to analyze the influences in the fixation of proximal femur fractures in a more physiologic way. The specific objectives were (i) to develop an embedding system that is independent of the individual geometry of the used human femora and still allows comparative results in different setups using common implants. Furthermore, a test protocol was developed that allows (ii) a loading of the specimen adapted to the original BW of the donor bones. Finally (iii), the direction of the loads was applied according to physiological patterns.

\section{Materials and methods}

\section{Load}

Bergmann et al. [2] described the dynamic orientation of the acting loads during normal walking with one step per second in the hip joint by the ab- and adduction and ante- and retroversion angles. These data of acting loads and motion pattern during normal walking are the bases for this in vitro setup. Figure 1A shows the described triplanar motion pattern of the acting load vector onto the femoral head surface during one step, represented to the center of the femoral head in a biplanar curve (Figure 1B). As a result, a representative idealized loading distribution through a linearization of these complex curve in a new coordinate system (here demonstrated by a $45^{\circ}$ rotation of the axes) and a temporal amplitude of the femoral movement can be taken (Figure 1B, simulated angles). Hence, the maximum angle peaks with corresponding load peaks were obtained (Figure 1B; physiologic and simulated load curve).

The load for each specimen pair was calculated by the individual BW. The load maximum was fixed with 2.8 times of the BW.

\section{Validation}

For validation of the test system, 12 paired human cadaveric femora from the anatomic institute were used. The probes showed no macroscopic deformation and previous trauma. The X-rays revealed no defects of the bones. The donor data of gender, BW, body height, and age were documented and were in a mean range for typical patients of hip fractures. The bone specimens were frozen at a minimum of $-20^{\circ} \mathrm{C}$ until $24 \mathrm{~h}$ prior to testing. In a standardized procedure, a defined A2.2 (AO fracture classification 3.2) fracture was set with a jigsaw. The Proximal Femoral Nail Antirotation (Synthes Inc., West Chester, PA, USA; Titanium $200 \mathrm{~mm} / 130^{\circ} / 10 \mathrm{~m}$ ) and the Gamma3 Nail (Stryker, Kalamazoo, MI, USA; Titanium $200 \mathrm{~mm} / 130^{\circ} / 10 \mathrm{~m}$ ) - two standard intramedullary implants - were used to show the usability of the test system. The implants were randomized according to a plan for the right and the left femora of one donor. The length of the lag screws or blades was adapted to the size of the neck and head of the
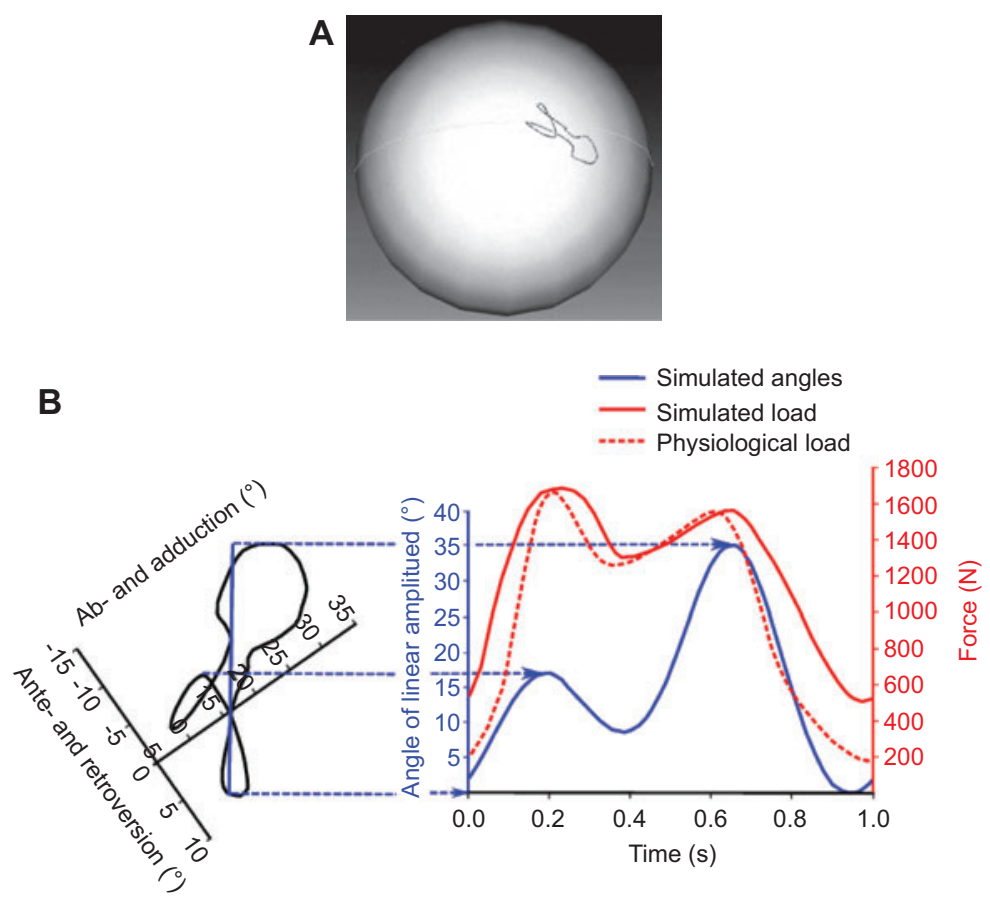

Figure 1 Motion patterns of the acting loads.

(A) On the surface of the femoral head during one step. (B) During normal walking in one step to the center of the femoral head by the abadduction and version angle. Linear extrapolation of the curve shows the relevant points in an amplitude-time graph with the corresponding simulated and physiological loads. 
individual femur. The osteosyntheses were implanted using the surgical instruments provided by the manufacturers and following the corresponding standard implantation technique. To achieve the exact position of the implant in the femur and especially in the head of the femur, a custom guiding arm was fixed on the original guiding instruments. The positions of the implants were documented by a pre- and post-test X-ray in two planes (anterior-posterior/lateral).

\section{Embedding}

To eliminate influences of individual geometries of human proximal femora, a special embedding system was designed. The specimens were embedded using cement (MOLDASTONE, Heraeus Kulzer GmbH, Hanau, Germany) with a compressive strength of $54 \mathrm{~N} / \mathrm{mm}^{2}$. The femoral head was embedded in a stainless steel artificial acetabulum, so that the center of the artificial acetabulum and the femoral head were congruent. The femoral shaft end was embedded in a conic aluminum form. While specimen embedding, the osteosyntheses were taken as a reference of the position. All dimensions of the exact femur and embedding foam position were referenced to the osteosyntheses design. Hence, the embedding position is determined through the implant position within the femur. The exact position of the implant is controlled by the company's guiding system and a custom guiding arm, which allows the implantation of the lag screw/ blade within $\pm 1 \mathrm{~mm}$ in the head of the femur. Thus, the horizontal axes are in alignment with the upper nail axes, and the angle in the frontal and sagittal plane is described through the implant axes, such that the nail and implant axes span a plane. For pretending the later femur position in the test rig, this plane encloses a $45^{\circ}$ angle to anterior-posterior direction. In addition, the distal and the proximal distances between the implant and the bone is not relevant for the results. The proximal femur has within this technique just the function as cladding material through which the applied load is inserted and deduced in the implant, respectively. Thus, all individual specimens with their different geometric and anatomic attributes are compensated with this special embedding method, and the influence on failure mechanisms is strictly reduced. Therefore, a better comparability in terms of reproducibility of results is guaranteed in all experiments.

\section{Load direction}

This movement is realized in the test rig by an electric motor that drives a linear bearing for the femoral movement via a newly developed custom device gearbox. The femur, positioned upside-down, is fixed over an embedding device at the shaft end in a pivotable linear bearing. Over a lever arm on the gearing mechanism, a distal detection system is applied. The horizontal movement effects through the fixed geometry and bearing disposition of the embedded femur a vertical amplitude to the applied movement. To each position of the linear guidance or detection of the femur, a calibrated spring delivers the corresponding force values. Therefore, the femoral head glides due to a steel calotte on three ball bearings as a low-friction simulation of the physiological hip joint (Figure 2). Through the variation of the spring stiffness, realistic experimental forces are simulated. A maximal load of $2.8 \mathrm{BW}$ with a course according to Figure 1B is applicable over admitted cycles. They are varying and are adjustable up to a manifold of the BW of the individual donor. The velocity of the gait simulation is continuously adjustable through the drive system. Especially the force peaks with their according gait angles are reproduced in the setup, respectively accurate (force-time-function).

\section{Data collection}

During all load cycles, a LabVIEW-based force measuring system continuously documented load peaks with a $50 \mathrm{kN}$ load cell (Instron, Norwood, MA, USA). In addition, the complete force progression during cycles was captured. For the acquisition of local deformations of the femur, a three-dimensional (3D)infrared tracking system (PCReex, Qualisys AB, Gothenburg, Sweden) was used. By rigid fixation of markers on the head of the femur and the implant, the relative movement between these two solid bodies can be calculated. The degree of varus collapse $\left(\propto_{\mathrm{CO}}\right)$ and rotation with respect to the implant axis $\left(\propto_{\text {Rot }}\right)$, but also migration direction of the relative movement between the tip of the lag screw and femoral head, was continuously measured as a function of applied loading cycles. Therefore, the absolute migration direction was separated into an axial $\left(\mathrm{S}_{\text {axial }}\right)$ and a cephalad $\left(\mathrm{S}_{\text {cephalad }}\right)$ component (Figure 3$)$.

\section{Results}

The calibration of the testing system via a standardized femur made out of stainless steel showed an accuracy and

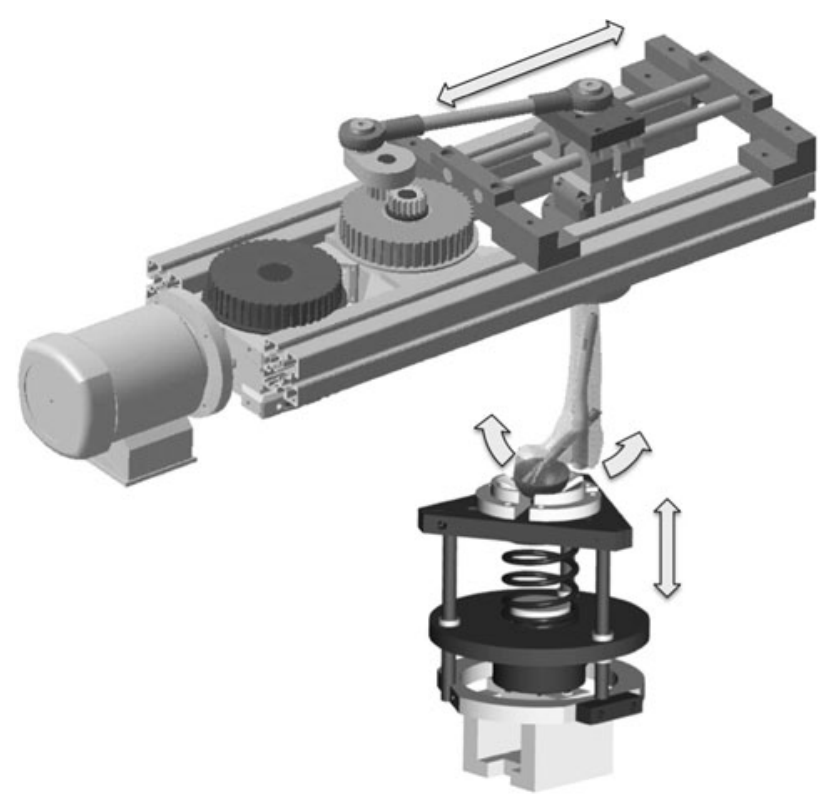

Figure 2 Schematic setup conception (drive and load application unit) including the main moving direction. 


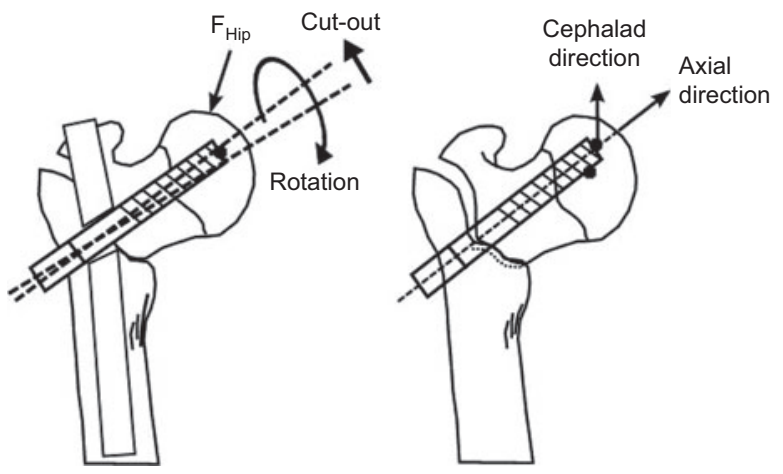

Figure 3 Migration pattern of osteosyntheses for angulation $\left(\propto_{\mathrm{CO}}\right)$, rotation $\left(\propto_{\text {Rot }}\right)$, and migration $\left(\mathrm{S}_{\text {axial }}\right)\left(\mathrm{S}_{\text {cephalad }}\right)$.

reproducibility for the load of $\pm 0.5 \mathrm{~N}$ and for the detection of the angle of $\pm 1^{\circ}$.

For angular movement, the varus collapse to cut out $\left(\propto_{\mathrm{CO}}\right)$ was the dominant failure mode, and only slight rotational angle shifts $\left(\propto_{\text {Rot }}\right)$ of the femoral head around the implant axis were observed independent of implant design. Angular displacements in varus direction were higher in specimens reinforced with screws.

After 1000 cycles, varus collapse angles to cut out of $\propto_{\mathrm{CO}}=0.29^{\circ} \pm 0.07^{\circ}$ for blade and $\propto_{\mathrm{CO}}=1.3^{\circ} \pm 1.19^{\circ}$ for screw were apparent (Figure 4).

For increasing load cycles, both $\propto_{\text {Rot }}$ and $\propto_{\text {CO }}$ increased (Figure 4). After 25,000 cycles, neck rotation reached a value of $\propto_{\text {Rot }}=1.7^{\circ} \pm 0.4^{\circ}$ for blade and $\propto_{\text {Rot }}=2.4^{\circ} \pm 0.3^{\circ}$ for screw. The angle of varus collapse to cut out had a greater magnitude with $\propto_{\mathrm{CO}}=4.8^{\circ} \pm 2.1^{\circ}$ for blade and $\propto_{\mathrm{CO}}=7.8^{\circ} \pm 3: 8^{\circ}$ for screw. There were no significant differences between the different implants in each load cycling group.

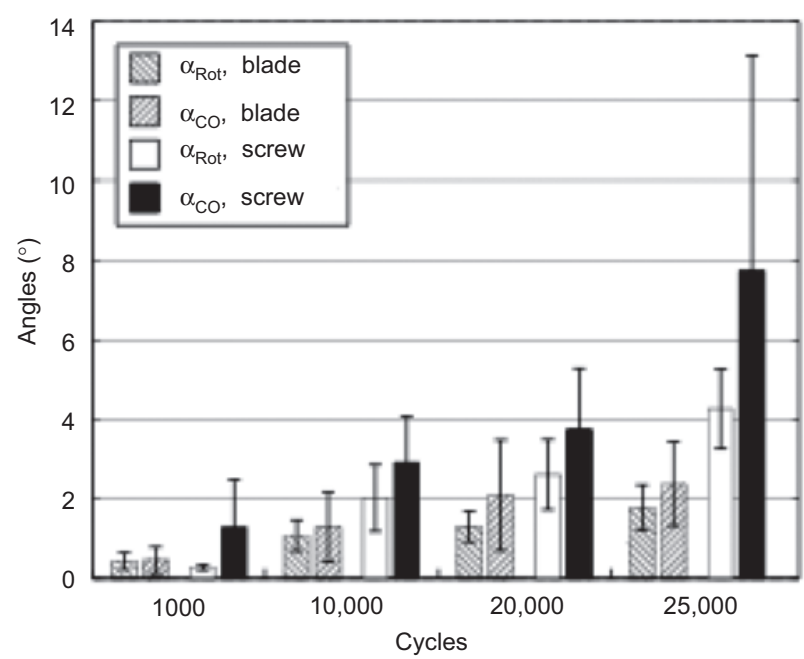

Figure 4 Comparison between blade $(n=12)$ and screw $(n=12)$ angle shifts: femoral head rotation around the implant $\left(\propto_{\text {Rot }}\right)$ and into varus collapse to cut out $\left(\propto_{\mathrm{CO}}\right)$.
The migration direction between the femoral head and the implant tip showed a tendency of a more cephalad than axial migration, also independent of implant design. Therefore, the cephalad migration had a consistent increase with higher loadings and reaches a value of $S_{\text {cephalad }}=1.69 \pm 0.29 \mathrm{~mm}$ for blade and $\mathrm{S}_{\text {cephalad }}=3.17 \pm 1.99 \mathrm{~mm}$ for screw (Figure 5) after 25,000 cycles. The axial migration had a slight slope for both implant designs over the whole testing (Figure 5) and a magnitude of $\mathrm{S}_{\text {cephalad }}=0.6 \pm 0.4 \mathrm{~mm}$ for blade and $\mathrm{S}_{\text {cephalad }}=0.5 \pm 0.3$ $\mathrm{mm}$ for screw after 25,000 cycles.

\section{Discussion}

In this project, a test system was developed to analyze osteosyntheses after hip fractures in a more physiologic way. The testing machine allows the clinically relevant mechanisms of the implant and bone interaction and their failure mechanisms to be observed. The new setup gives evidence about the anchorage stability of different implant types under a dynamic gait motion pattern.

The developed test system uses a protocol that allows a percentile loading of the specimen according to the original $\mathrm{BW}$ of the donor bones. This gives an optimized interindividual comparison of the specimen results because the load is adapted to the individual bone.

The direction of the applied loads was adapted to physiological patterns, and the results of the study by Bergmann et al. were used to replicate the loading curve for the gait simulation [2]. In particular, due to the simulated hip joint in the test system, a multiplanar movement (migration and rotation) of the probe is observed, which is up to now not reported in the literature. As a critical point, the loss of the stabilization by muscle and tendons can be seen. In the actual literature, there is no published testing system for the femur with muscle or tendon stabilization.

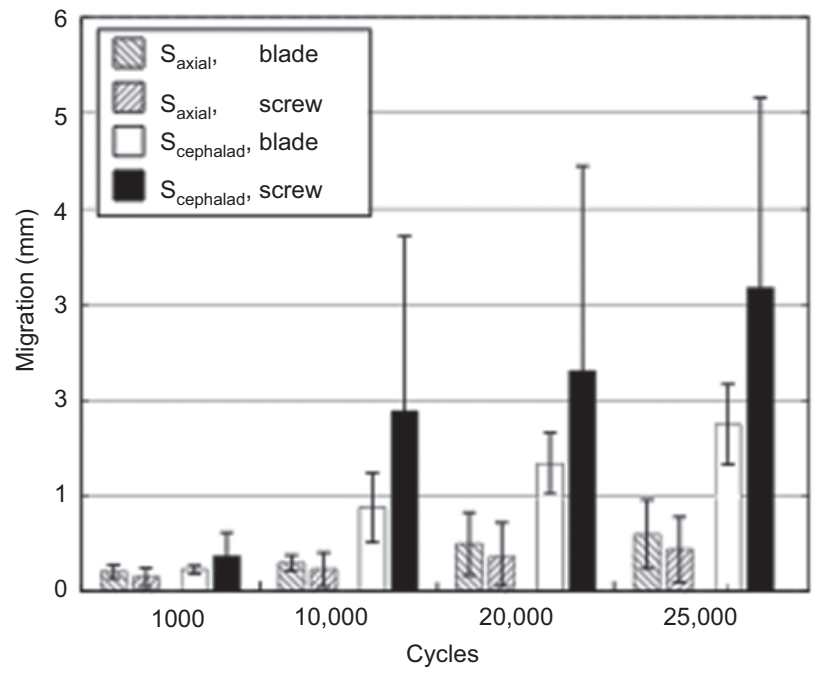

Figure 5 Comparison between blade $(\mathrm{n}=12)$ and screw $(\mathrm{n}=12)$ migration: in axial $\left(\mathrm{S}_{\text {axial }}\right)$ and cephalad $\left(\mathrm{S}_{\text {cephalad }}\right)$ directions. 
Human bone specimens show a large individual variety in geometry; therefore, a uniform way to set a fracture and to embed the specimen is necessary.

An embedding system was designed to consistently fix different implants in cadaveric bones without the influence of the individual geometry of the human bones. According to the literature, the A2.2 fracture type (AO fracture classification 3.2 ) is the most commonly seen unstable intertrochanteric femur fracture $[11,14,17,19]$ and is used in several biomechanical investigations [12].

An interesting and novel result of our model is the large amount of rotation and the characteristic failure path for each specimen. Independent of the implant type, the stages of failure were: (i) rotation of the head, (ii) migration of the implant, and (iii) varus collapse. No difference in rotation, migration, or varus collapse has been documented between the implant types until 1000 load cycles. A difference between the used implants could be observed after 25,000 load cycles but showed no statistically significant difference.

The rotation between the different screws and blades and the femoral head under load is described by Sommers et al. [20] for decentral position of the implant in the femoral head. To minimize this effect, we positioned the lag screw in a central-central position in the head by a custom guiding instrument. The documented rotation is a result of the multiplanar loading of the hip.

In consent with other biomechanical investigations on hip fracture osteosyntheses, the multiplanar dynamic test is the most promising way to investigate the complex load situation at the hip joint under physiological gait simulation. In particular, rotation and migration movements could not be observed in former uniplanar static or dynamic tests but are clinically relevant. Similar results were found by the group of Ehmke with a multiplanar dynamic test, but in isolated femoral head and neck specimen [6].

In contrast to other studies on migration behavior in the literature, which use discontinuous measuring devices for migration measurement, the deformation data of this study were continuously registered by optical infrared measuring with fixed marker systems, i.e., by measuring the rigid body motion and interaction between the implant and the femoral head. The continuous data collection describes an exact motion map of the migration and rotation.

Rotation about the lag screw is not typically considered as a failure mechanism for hip fractures. However, a study by Mils and Horne describes $30^{\circ}$ rotations about the femoral neck during the insertion of a lag screw and postulates that these rotations may not be detectable on standard radiographs, indicating that the intraoperative ability to detect rotation is poor [16]. The rotation seen in our specimens was always $<10^{\circ}$. Lustenberger et al. published that $12 \%$ of pertrochanteric fractures undergo rotation as they collapse [15].

In clinical practice, implant failures are evaluated on twodimensional-radiographs and show various collapses to cut out and femoral head rotation around the implant. However, a differentiation between "cut out" and "cut through" is not yet published. The cut through can be described as an axial migration of the lag screw or blade through the center of the femoral head cortex as it is described by the "Z-effect" phenomenon [21]. In contrast, the cut out is a varus migration through the superior or cephalad part of the femoral head.

Within this setup, both angle shifts, rotation, and varus collapse could be seen due to the optical 3D-infrared marker system, but also a distinction of the migration direction between the implant and the femoral head fragment in axial $\left(\mathrm{S}_{\text {axial }}\right)$ and cephalad $\left(\mathrm{S}_{\text {cephalad }}\right)$ directions could be documented. Therefore, the varus collapse to cut out and cephalad migration to cut through were the predominant failure modes independent of implant design.

On purpose, in this first evaluation of the testing system to investigate the effects of physiologic loads, a "worst-case" scenario was not simulated.

The selected osteotomy represents an unstable fracture according to AO classification. Due to a bony interface in the fracture gap produced using a saw and a central implant position within the femoral head, the induced rotational moment was small. After an initial rotation, the femoral head fragment slides due to the gliding mechanism in the nail toward the shaft fragment and gets stabilized by impaction. As varus collapse begins and the implant starts to migrate within the femoral head, a more eccentric position with a greater rotational moment could result in a slight rotational increase with load cycles as also described by Al-Munajjed et al. [1] and Sommers et al. [20].

The bony support between the head and the shaft fragments also constrains the migration in axial direction, so that for both implants, cephalad migration was dominant. Hence, the helical blade showed a higher resistance compared to the used screw. The study of Karich et al. [12] also describes hip screw and blade migration under near-physiological conditions. Surrogate specimens were used to simulate the worst-case scenario without bony support. The failure criterion was defined as the specific load at which plastic deformations reached a value of $2 \mathrm{~mm}$. According to the results, the hip screw migrated predominantly in the cephalad direction toward the proximal edge of the femoral head and less in their axial direction, whereas helical blades showed a small cephalad migration but a more distinctly axial direction into the femoral head. This different migration behavior could explain the distinct magnitude of cephalad migration between helical blade and screw geometry. The architecture of the trabecular network appears to be inhomogeneously oriented. The architecture of the trabecula network follows the trajectories of the main loading direction, so that bending is minimized. The fatigue strength is highly anisotropic for cancellous bone [23]. The relationship between the cyclic deformation and fatigue behavior and axis of loading of cancellous bone has been described for human trabecular bone and uniaxial cyclic compression [4]. Already small deviations of the specimens' axes from the main physiological bone axes result in a largely reduced lifetime [4].

The axial migration direction is more aligned with the main physiological axis, whereas the cephalad direction is considered to load cancellous bone in a more off-axis angle direction. The migration direction determines the stability of the implant-bone compound due to the anisotropy of cancellous bone. Thus, a migration of the implant in a more cephalad 
direction is supposed to be less stable. Like in all experimental fatigue studies on bone up to now, no biological effects in terms of remodeling processes could be included in this study, and their influence remains unclear.

\section{Clinical relevance}

Clinical complications due to implant failure are caused by damage of the trabecular structure in the bone due to microcracking, through implant-induced stress concentration fractures or age-related fragility fractures. The high dependency of fatigue lifetime on the axis of loading may have direct clinical consequences. Hence, fracture treatment through implants may preserve the physiological load axis. Thus, implant anchorage should lead to mostly on-axis loading, and regions of low structural anisotropy should be preferred. This study does support this concept with a new migration model that enables showing the large influence of the axis of loading on the fatigue behavior of cancellous bone.

Both implants are successfully used in clinical practice, but in vivo studies have consistently failed to find out significant differences between implant designs. This in vitro study on cadaveric specimens enabled simulation of clinical failure and showed a distinction between implant designs in terms of biomechanics. Thus, screw design showed less resistance under near-physiological loading against cephalad migration compared to helical blade. This should be considered when using these kinds of implants in osteoporotic patients with intertrochanteric fractures. Furthermore, results of in vitro migration investigations under near-physiological loading are essential for continuous improvements of implants especially in the preclinical design of the anchoring system in the cancellous bone. The results could give a better understanding of the clinical failure mechanisms and can be the scientific basis for future implant optimization.

\section{Conclusions}

The developed embedding system is independent of the individual geometry of the used human femur and allows comparative results in different setups using common implants.

Furthermore, a test protocol was developed, which allows loading of the specimen according to the original $\mathrm{BW}$ of the donor bones.

The system is able to apply the direction of the loads according to physiological patterns.

The new testing system gives evidence about the anchorage stability of different implant types under dynamic gait motion pattern. Therefore, the cut out model and the migration directions showed a tendency between different implant types.

\section{Acknowledgements}

The biomechanical tests were part of a sponsored program to investigate the implant stability at the proximal femur by the Synthes AG.

\section{References}

[1] Al-Munajjed AA, Bachmeier S, Hammer J, Lenich A. Biomechanical characterisation of osteosyntheses for proximal femur fractures: helical blade versus screw. Stud Health Technol Inform 2008; 133: 1-10.

[2] Bergmann G, Deuretzbacher G, Heller M, et al. Hip contact forces and gait patterns from routine activities. J Biomech 2001; 34: 859-871.

[3] Braithwaite RS, Col NF, Wong JB. Estimating hip fracture morbidity, mortality and costs. J Am Geriatr Soc 2003; 51: 364-370.

[4] Dendorfer D, Maier HJ, Taylor D, Hammer J. Anisotropy of the fatigue behaviour of cancellous bone. J Biomech 2008; 41: 636-641.

[5] Den Hartog BD, Bartal E, Cooke F. Treatment of the unstable intertrochanteric fracture. Effect of the placement of the screw, its angle of insertion, and osteotomy. J Bone Joint Surg Am 1991; 73: 726-733.

[6] Ehmke LW, Fitzpatrick DC, Krieg JC, Madey SM, Bottlang M. Lag screws for hip fracture fixation: evaluation of migration resistance under simulated walking. J Orthop Res 2005; 23 : 1329-1335.

[7] Evans EM. The treatment of trochanteric fractures of the femur. J Bone Joint Surg 1949; 31-B: 190-203.

[8] Frerichmann U, Raschke MJ, Stöckle U, Wöhrmann S, Lohmann R. Proximal femoral fractures in the elderly. [Data from health insurance providers on more than 23 million insured persons part 2] [Article in German]. Unfallchirurg 2007; 110: 610-616.

[9] Haynes RC, Pöll RG, Miles AW, Weston RB. An experimental study of the failure modes of the gamma locking nail and AO dynamic hip screw under static loading: a cadaveric study. Med Eng Phys 1997; 19: 446-453.

[10] Haynes RC, Pöll RG, Miles AW, Weston RB. Failure of femoral head fixation: a cadaveric analysis of lag screw cut-out with the gamma locking nail and AO dynamic hip screw. Injury 1997; 28: 337-341.

[11] Johnell O, Kanis JA. An estimate of worldwide prevalence, mortality and disability associated with hip fracture. Osteoporos Int 2004; 15: 897-902.

[12] Karich B, Oldenburg GV, Simon B, Bauer C. A new test method to determine the cut-out behaviour of hip screws. J Biomech 2006; 39: S525.

[13] Kauffman JI, Simon JA, Kummer FJ, et al. Internal fixation of femoral neck fractures with posterior comminution: a biomechanical study. J Orthop Trauma 1999; 13: 155-159.

[14] Lenich A, Mayr E, Rüter A, Möckl C, Füchtmeier B. First results with the trochanter fixation nail (TFN): a report on 120 cases. Arch Orthop Trauma Surg 2006; 126: 706-712.

[15] Lustenberger A, Bekic J, Ganz R. [Rotational instability of trochanteric femoral fractures secured with the dynamic hip screw. A radiologic analysis] [Article in German]. Unfallchirurg 1995; 98: 514-517.

[16] Mils HJ, Horne G. Displacement of subcapital fractures during internal fixation: a real problem? Aust N Z J Surg 1989; 59: 249-251.

[17] Parker MJ, Handoll HH. Gamma and other cephalocondylic intramedullary nails versus extramedullary implants for extracapsular hip fractures in adults. Cochrane Database Syst Rev 2005; (Online) (4): CD000093.

[18] Schürch MA, Rizzoli R, Mermillod B, Vasey H, Michel JP, Bonjour JP. A prospective study on socioeconomic aspects of fracture of the proximal femur. J Bone Miner Res 1996; 11: 1935-1942. 
[19] Smektala R, Hupe K, Paech S. [Trochanteric femur fractures - analysis of external quality assurance within a comprehensive survey] [Article in German]. Z Ärztl Fortbild Qualitatssich 2005; 99: 537-545.

[20] Sommers MB, Roth C, Hall H, et al. A laboratory model to evaluate cutout resistance of implants for pertrochanteric fracture fixation. J Orthop Trauma 2004; 18: 361-368.

[21] Strauss EJ, Kummer FJ, Koval KJ, Egol KA. The "Z-effect" phenomenon defined: a laboratory study. J Orthop Res 2007; 25: $1568-1573$.
[22] Wu CC, Shih CH, Lee MY, Tai CL. Biomechanical analysis of location of lag screw in treatment of unstable intertrochanteric fracture. J Trauma 1996; 41: 699-702.

[23] Yang G, Kabel J, van Rietbergen B, Odgaard A, Huiskes R, Cowin SC. The anisotropic Hooke's law for cancellous bone and wood. J Elast 1999; 53: 125-146.

Received July 28, 2010; accepted February 14, 2012 